\title{
Single-Molecule Analysis of Translational Dynamics
}

\author{
Alexey Petrov ${ }^{1}$, Jin Chen $^{1,2}$, Seán $\mathrm{O}^{\prime}$ Leary $^{1}$, Albert Tsai ${ }^{1,2}$, and Joseph D. Puglisi ${ }^{1}$ \\ ${ }^{1}$ Department of Structural Biology, Stanford University School of Medicine, Stanford, California 94305-5126 \\ ${ }^{2}$ Department of Applied Physics, Stanford University, Stanford, California 94305-4090 \\ Correspondence:puglisi@stanford.edu
}

Decades of extensive biochemical and biophysical research have outlined the mechanism of translation. Rich structural studies have provided detailed snapshots of the translational machinery at all phases of the translation cycle. However, the relationship between structural dynamics, composition, and function remains unknown. The multistep nature of each stage of the translation cycle results in rapid desynchronization of individual ribosomes, thus hindering elucidation of the underlying mechanisms by conventional bulk biophysical and biochemical methods. Single-molecule approaches unsusceptible to these complications have led to the first glances at both compositional and conformational dynamics on the ribosome and their impact on translational control. These experiments provide the necessary link between static structure and mechanism, often providing new perspectives. Here we review recent advances in the field and their relationship to structural and biochemical data.

T⿱宀⿻三丨日巾 anslation and its regulation are intrinsically dynamic processes. In all organisms, to initiate translation, ribosomes must assemble from isolated subunits and an initiator transfer RNA (tRNA) on a messenger RNA (mRNA) at a specific start codon to establish a reading frame; protein factors guide this process. Elongation occurs through selection by the ribosome of cognate aminoacyl tRNAs, subsequent positioning of tRNAs for peptide bond formation chemistry, and movements of the tRNAs and mRNAs with respect to the codon (translocation). The directional process is iterative until termination at a stop codon, where the protein chain is released, and the ribosomal particle disassembled and recycled. Multiple ribosomes form higher-order polysomes on a single mRNA, with their own intrinsic dynamics.

Dynamics are central to the mechanism and control of translation. Here we explicitly define dynamics as time-dependent changes in either composition or conformation of the translational machinery. Conformational dynamics in chemical systems are governed by an array of processes with vastly different timescales. Generally, dynamic processes become slower as they involve larger numbers of atoms. These range from electronic motions (timescale $10^{-14} \mathrm{sec}$ ), bond vibrations $\left(10^{-13}-10^{-12} \mathrm{sec}\right)$, through protein side chain or nucleic acid base/sugar local conformational changes $\left(10^{-11}-10^{-6} \mathrm{sec}\right)$, to larger conformational rearrangements (domain

Editors: John W.B. Hershey, Nahum Sonenberg, and Michael B. Mathews

Additional Perspectives on Protein Synthesis and Translational Control available at www.cshperspectives.org

Copyright (C) 2012 Cold Spring Harbor Laboratory Press; all rights reserved; doi: 10.1101/cshperspect.a011551

Cite this article as Cold Spring Harb Perspect Biol 2012;4:a011551 
A. Petrov et al.

movements, etc.; $\left.10^{-6}-10^{2} \mathrm{sec}\right)$ that are often functionally cooperative. Compositional dynamics are determined by bimolecular association and dissociation rate constants: bimolecular arrival rates for ligands are governed by intermolecular collision frequencies, electrostatic interactions, and proper binding orientations for productive binding events, whereas dissociation rates are governed by energy barriers for dissociation of noncovalent intermolecular interactions.

Fluctuations in molecular conformation and composition must be harnessed by the ribosome for accurate and rapid translation. The timescales of these dynamic changes dictate the overall rates of translation initiation and elongation: $0.2-0.5$ initiation events/ sec and elongation rates of 20-40 amino acids/sec in vivo, 1-5 amino acids/sec in vitro (Dennis and Bremer 1974a,b; Underwood et al. 2005). The ribosome uses external sources of free energy during translation-ATP hydrolysis during eukaryotic scanning, GTP hydrolysis by initiation, elongation and termination factors, and peptide bond formation. The free energy released by these irreversible reactions is used to drive the fidelity of initiation and elongation and the directional movement of the ribosome during both processes. The ribosome is thus a molecular motor.

The link between ribosome and ligand dynamics and the control of protein synthesis remains a key mystery of translation. The past decade has witnessed the three-dimensional structures of prokaryotic, archaeal, and eukaryotic ribosomes at atomic resolution. How factors, tRNA, and ligands interact with the ribosome has been revealed by cryo-electron microscopy (cryo-EM) (at lower 6-12 $\mathrm{\AA}$ resolution) and X-ray diffraction studies (as low as $2.5 \AA$ for $30 \mathrm{~S}$ from Thermus thermophilus [Kurata et al. 2008] and $2.4 \AA$ for 50 S from Haloarcula marismortui [Ban et al. 2000]). These structures have shown how GTPase factors engage with the $70 \mathrm{~S}$ ribosomes at a conserved factor-binding site on the large subunit to mediate GTPase activity and subsequent conformational changes. Another key observation of early cryo-EM and more recent structural studies is that the ribosome adopts two general intersubunit conformations, related by a $6^{\circ}$ rotation of the two subunits (Valle et al. 2003; Schuwirth et al. 2005; Agirrezabala et al. 2008; Zhang et al. 2009; Fischer et al. 2010; Dunkle et al. 2011). Peptide bond formation leads to a counterclockwise rotation of the small subunit with respect to the large subunit, and EF-G in the GTP form binds to this state. The peripheral domain L1 region of the ribosome was observed structurally to change its state in correlation with the two ribosomal conformations (Valle et al. 2003; Schuwirth et al. 2005; Agirrezabala et al. 2008), suggesting a coupling of domains within the ribosome. The intersubunit conformation of the ribosome was also correlated by EM to the relative orientations of tRNAs: In the nonrotated state (locked conformation), tRNAs are observed in the classical $\mathrm{P}$ site and $\mathrm{A}$ site, whereas in the rotated state (unlocked conformation) the tRNAs are in the Noller hybrid states with the $3^{\prime}$ ends of the tRNAs moved to the $\mathrm{E}$ and $\mathrm{P}$ sites and their respective anticodons in the P and A sites (Agirrezabala et al. 2008). These static structural views suggested a further correlation of tRNA and ribosome conformation during translation.

The structural snapshots and prior biochemical studies are suggestive of dynamics during translation, yet experimental methods with resolution in real time are required to observe them directly. Here we focus on singlemolecule methodologies that have provided an unprecedented view into the dynamics of prokaryotic translation. In the future, the same techniques can be applied to the study of eukaryotic translation dynamics.

\section{WHY SINGLE-MOLECULE APPROACHES?}

Dynamics have been traditionally measured using bulk methods, with signals sensitive to dynamic changes for a large collection of molecules. These signals, such as emission from a fluorescent dye, must be sensitive to conformational or compositional changes of the system as it evolves in time. In the case of translating ribosomes, problems arise in synchronizing a large collection of molecules to detect a change 
in the bulk signal for a specific process. Imagine, for example, a signal that changes on tRNA binding to the A site. In order to detect a change in this signal, the system must be synchronized such that all tRNAs are unbound at the start of the measurement, at which point reaction usually started through rapid mixing. This ensures that the observed time-dependent fluorescence change reports only on the approach to equilibrium from the unbound state, and from this signal kinetic information can be extracted. However, if we want to look at a subsequent tRNA-binding event, we would have to pause the evolution of the system, remix the reagents, and repeat the measurement. In short, dynamics cannot be measured in real time during multiple rounds of elongation. This need for synchronization is a fatal limitation of bulk kinetic investigations to probe multistep dynamic systems.

Single-molecule experiments allow direct measurement of dynamics without the need for synchronization. Commonly used organic fluorophores such as rhodamine derivatives and cyanine dyes emit sufficient numbers of photons to be readily detected with modern cameras at millisecond time resolution. To distinguish weak single-fluorophore fluorescence from background illumination and noise, various techniques such as total internal reflection fluorescence microscopy (TIRF) and zero-mode waveguides (ZMWs) are utilized (Ha 2001; Levene et al. 2003). The rapid diffusion of fluorophores in solution limits observation of the free molecules, because on the timescale of data acquisition a labeled molecule leaves the illumination volume before emitting enough photons to be observed.

The problem of diffusion can be turned into an advantage by spatially constraining the system through surface immobilization - in translation experiments this is most often accomplished using biotin-streptavidin interactions to bind mRNAs or ribosomes to an optically transparent surface. Let us take a simple example of the bimolecular binding event of a dyelabeled tRNA to an immobilized ribosome. In the single-molecule fluorescence experiment, the freely diffusing unbound tRNA is invisible and binding of the tRNA to an immobilized ribosome leads to a burst of observed fluorescence, as the fluorophore, emitting a large number of photons, is now localized within a small observation volume, as opposed to freely diffusing in solution. Binding of multiple tRNAs is manifested at the single-molecule level as a series of fluorescence bursts and interburst delays. The observed burst lifetimes and delay times yield time constants that are reciprocal to the rate constants for dissociation and association, respectively.

The power of this approach is revealed when we observe binding of a second tRNA labeled with a differently colored dye. The single-molecule analysis of this multicolor experiment would reveal the relative arrival time for the two different tRNAs, by the time interval between the fluorescence bursts, as well as how long the two bound tRNAs overlapped on the same ribosome, as a duration of the bursts overlap. These results can be obtained directly from the data without the need to synchronize the ribosomes during the experiment. "Postsynchronization" in silico yields the relative timing of two or more events. This procedure amounts to aligning all single-molecule traces from an experiment using a single common event that defines the new zero point along the time axis of each trace. Further, in the case of a heterogeneous system, single-molecule traces may be sorted and each subset analyzed independently. Therefore the order of binding events, kinetics of the subsequent binding and dissociation events, and average overlap time of occupancy by multiple ligands can be measured independent of system complexity, and correlations between dynamic events in multistep, heterogeneous systems can be observed directly with a single-molecule approach.

Conformational dynamics are also readily investigated using single-molecule fluorescence. The main tool for this application is Förster resonance energy transfer (FRET), which involves energy transfer between a donor and acceptor dye through coupling of transition dipoles. The efficiency of energy transfer depends on $1 / R^{6}$ in which $R$ is the interdye distance, as well as on the spectral overlap of the two dyes and on dye orientation terms. For Cy3 and Cy5 
A. Petrov et al.

dyes, commonly used dyes used in these experiments, the efficiency of FRET varies from 1 (e.g., all emissive energy from $\mathrm{Cy} 3$ is transferred to Cy5) at distances below about $20 \AA$, to 0 at distances above $80 \AA$. Thus FRET is highly sensitive to distance changes in the region of $30-$ $60 \AA$, well suited for investigation of the ribosome ( $250 \AA$ in diameter).

FRET represents a high time-resolution probe of biomolecular conformation. In singlemolecule FRET (smFRET), donor and acceptor dye emissions are measured simultaneously for individual molecules, and these intensities are converted to FRET through the equation $E_{\text {obs }}=I_{\text {acceptor }} /\left(I_{\text {donor }}+I_{\text {acceptor }}\right), \quad$ in which $I_{\text {donor }}$ and $I_{\text {acceptor }}$ are fluorescence intensities of the donor and acceptor dyes, correspondingly. Changes in interdye distance are revealed by anticorrelated changes in donor and acceptor intensities. Molecular conformation can be monitored by smFRET with millisecond time resolution, limited by fluorophore brightness and camera sensitivity. smFRET has been a powerful tool to explore ribosomal and ligand dynamics during translation, as outlined below.

In addition to dynamics, single-molecule methods can directly measure forces generated by molecular motors and mechanical stability of molecular complexes. Optical traps are instrumental in revealing the mechanism of motor proteins and in mechanistic studies of translation. Optical traps can apply constantly increasing force to the point of complex rupture to test mechanical stability of the complexes, thus directly reporting on the tensile strength of the intermolecular interactions, such as those between mRNA and the ribosome. Alternatively, the trap can be employed as a molecular ruler to track relative movement of the two components in the complex, for example, traveling of the ribosome along mRNA. Optical tweezers also permit application of an intermediate assisting or hindering force to the molecular motor, thus allowing elucidation of the mechanism of the motor mobility. These experiments are only possible at the single-molecule level. Modern optical traps allow distance measurement at the angstrom level of resolution and application of forces in the range of tens of piconewtons, with subpiconewton precision. The ribosome reads mRNA in 1.3-nm-long triplets and requires up to $20 \mathrm{pN}$ to dislocate from mRNA, thus it is suitable for study with single-molecule force methods.

Single-molecule experiments must be tackled with care and diligence. The approach, by its very sensitivity, is fraught with potential artifacts. Surface immobilization can perturb behavior of biological systems, and nonspecific surface interactions can interfere with singlemolecule measurements. Biologically relevant signals are convoluted with photophysical and photochemical artifacts due to high-intensity illumination-blinking, photobleaching, and photodamage limit single-molecule measurements and must be addressed by use of specific dyes, removal of molecular oxygen, and addition of chemical agents to improve dye behavior. The challenging and time-consuming nature of the single-molecule approach limits throughput. As a result, single-molecule experiments should be used in conjunction with conventional kinetic and molecular biology methods to address biological questions. Despite these limitations, the past decade has seen single-molecule data contributing deeply to our understanding of translation.

\section{TRANSLATION INITIATION}

The goal of translation initiation is to select the mRNA, recognize the correct start codon, and assemble an elongation-competent $70 \mathrm{~S}$ particle with an initiator tRNA in the $\mathrm{P}$ site. In prokaryotes initiation is guided by three initiation factors: IF1, the GTPase IF2, and IF3. The mRNA is directly recruited to the $30 \mathrm{~S}$ subunit via interactions between the mRNA Shine-Dalgarno sequence and a complementary sequence in the 16S ribosomal RNA (rRNA). The Shine-Dalgarno sequence is located 5-9 bases upstream of the AUG start codon, causing the start codon to be positioned correctly into the P site. IF2 and initiator tRNA are recruited to the $30 \mathrm{~S}$ subunit to form a $30 S$ preinitiation complex. IF2 then promotes joining of the $50 \mathrm{~S}$ ribosomal subunit. Formation of the 70S initiation complex triggers rapid GTP hydrolysis by IF2. The GDP-bound 
form of IF2 has lesser affinity for the 70 S ribosome and rapidly dissociates from the ribosome leaving the $70 \mathrm{~S}$ particle ready for the first round of elongation (Antoun et al. 2003).

\section{ORDER OF IF2 AND INITIATOR tRNA ARRIVAL}

The timing of key initiation events is crucial for translational control, although much of it remains unclear. It is a common prejudice in the literature that IF2 recruits initiator tRNA to the ribosome, but the experimental evidence is slim. The original biochemical studies showed that IF2 stabilizes tRNA in the 30 S preinitiation complex, but did not elucidate the order of ligand recruitment, leading to conflicting hypotheses (Lockwood et al. 1971; Wu et al. 1996; Wu and RajBhandary 1997). There are multiple possible arrival mechanisms: One of the ligands may arrive first, recruiting or permitting binding of the second one; both ligands may arrive simultaneously; or the order of arrival may be stochastic.

Although recent experiments suggested that IF2 and fMet-tRNA ${ }^{\text {fMet }}$ bind sequentially to the 30S subunit (Milon et al. 2010), IF2(GTP) also forms a weak complex with the tRNA $\left(K_{\mathrm{D}} \sim\right.$ $1 \mu \mathrm{M}$ ) (Lockwood et al. 1971; Petersen et al. 1979; Wu and RajBhandary 1997; Spurio et al. 2000; Milon et al. 2010), potentially allowing simultaneous binding of the two molecules. Tsai et al. (2012) used a ZMW-based singlemolecule approach utilizing fluorescently labeled IF2 and tRNA ${ }^{\mathrm{fMet}}$ to determine whether IF2 and tRNA binding is simultaneous, sequential, or random. The mixture of fMet-(Cy3)tRNA $^{\text {fMet }}$, Cy5-IF2, and Cy3.5-50S was delivered to immobilized Alexa488-30S. The appearance of a stable $50 \mathrm{~S}$ signal was used to identify productive tRNA- and IF2-binding events. The relative timing of IF2 and tRNA ${ }^{\text {fMet }}$ arrival to the ribosome was directly observed by single-molecule analysis (Fig. 1).

Without IF1 and IF3 at low concentrations of IF2 and the initiator tRNA (20 nM each) the tRNA arrives first in $65 \%$ of the initiation events, IF2 arrives first in $30 \%$, and in only $5 \%$ of cases do both IF2 and tRNA arrive simultaneously. The presence of IF1 and IF3 shifts the arrival order, with $50 \%$ of ribosomes having IF2 arriving before $\mathrm{tRNA}^{\mathrm{fMet}}, 40 \%$ having $\mathrm{tRNA}^{\mathrm{fMet}}$ arriving before IF2, and 10\% showing simultaneous arrival. This is consistent with IF1 and IF3 destabilizing tRNA ${ }^{\text {fMet }}$ in the 30S PIC (Antoun et al. 2006) and increasing the affinity of IF2 to the $30 \mathrm{~S}$ ribosomal subunit in the absence of initiator tRNA, leading to a higher ratio of molecules in which IF2 arrives first in their presence (Lockwood et al. 1972; Caserta et al. 2006). Increasing IF2 and tRNA ${ }^{\mathrm{fMet}}$ concentrations to $1 \mu \mathrm{M}$ raised the fraction of ribosomes showing simultaneous arrival to $45 \%$, whereas lowering the fraction of IF2 arriving first to $35 \%$ and the fraction of $\mathrm{tRNA}^{\mathrm{fMet}}$ arriving first to $10 \%$. The increase in simultaneous arrival with concentration of ligands suggests that at higher concentrations, a significant fraction of the tRNA and IF2 arrive as IF2-tRNA complexes. Thus, the order of IF2 and initiator tRNA arrival does not strictly follow a defined sequence, and is greatly affected by ligand concentrations and the presence of other initiation factors. Although at low concentrations the order of arrival is stochastic, in the presence of IF1 and IF3 and at high ligand concentrations simultaneous arrival may be a more common mechanism.

The observed dependence of the IF2 and tRNA recruitment on the presence of initiation factors and reaction conditions may explain the disagreement in results obtained by different groups. The recent observation by Milon et al. (Milon et al. 2010) suggests that IF2 binds first to the $30 \mathrm{~S}$ subunit and then recruits tRNA. These experiments were conducted in $20 \mathrm{mM}$ $\mathrm{MgCl}_{2}$, and $0.25 \mathrm{mM} \mathrm{GTP}$, whereas single-molecule measurements were performed at $5 \mathrm{mM}$ $\mathrm{MgCl}_{2}$, and $4 \mathrm{mMGTP}\left(\sim 1-2 \mathrm{mM}\right.$ free $\left.\mathrm{Mg}^{2+}\right)$. The IF2-tRNA complex is destabilized by high $\mathrm{Mg}^{2+}$ (Majumdar et al. 1976; Sundari et al. 1976; Spurio et al. 2000). Therefore the difference in $\mathrm{Mg}^{2+}$ concentrations could be a reason why Milon et al. have not observed simultaneous arrival of tRNA and IF2, whereas low magnesium conditions in single-molecule experiments facilitated formation of the IF2 and tRNA complex. The total concentration of magnesium in Escherichia coli is in the range of 50$200 \mathrm{mM}$ with most of this being bound to 
A. Petrov et al.
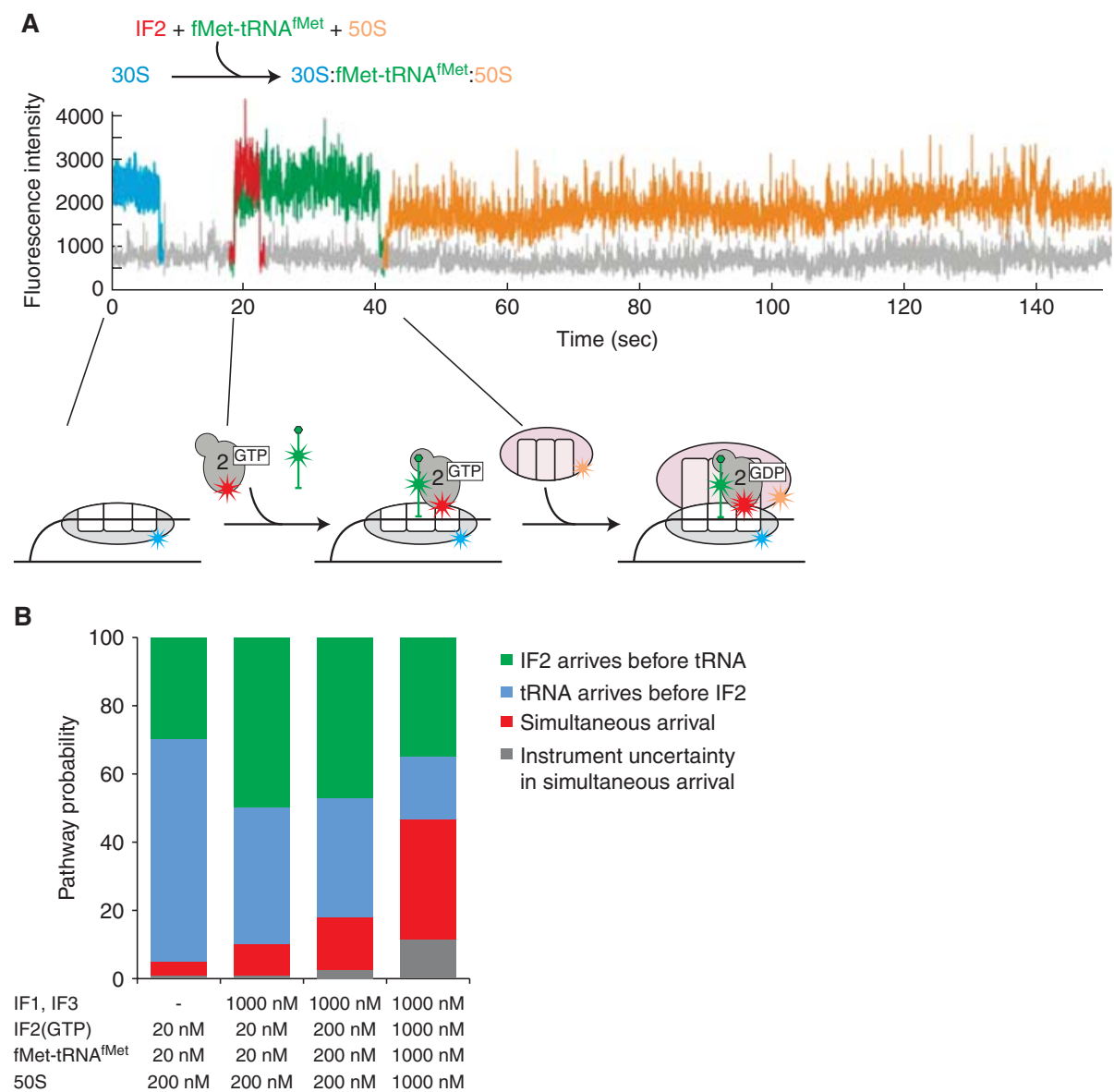

Figure 1. Timing of IF2 and tRNA arrival. (A) Example trace of timing of IF2 and initiator tRNA binding. 30SmRNA initiation complexes were immobilized in zero-mode waveguide (ZMW) wells. Fluorescent dyes are shown as stars. The presence of single $30 \mathrm{~S}$ complexes was identified by Alexa 488 fluorescence of labeled small ribosomal subunits, which photobleaches rapidly on start of observation due to the short photobleaching lifetime of the Alexa488 dye. Cy5-labeled IF2, Cy3-labeled fMet-tRNA ${ }^{\mathrm{fMet}}$, and Cy3.5 50S subunits allow direct observation of molecular events during initiation. In this example trace, IF2 and tRNA arrive simultaneously and then IF2 and tRNA signals disappear due to photobleaching as both ligands are required for subsequent stable $70 \mathrm{~S}$ complex formation. The initiation is finalized by stable arrival of $50 \mathrm{~S}$ at an $\sim 42 \mathrm{sec}$ time point. The order of arrival was directly determined to a single frame precision $(33 \mathrm{msec})$ by the sequence of the fluorescent pulses. (B) Observed ratios of $30 \mathrm{~S}$ preinitiation complex (PIC) formation pathways at different ligand concentrations. The order of arrival of labeled IF2 and initiator tRNA for each ribosome was observed and the ratios of possible arrival pathways at different ligand concentrations are plotted. (Modified, with permission, from Tsai et al. 2012.)

proteins or in chelates with anionic metabolites. The free $\mathrm{Mg}^{2+}$ concentration was estimated to be between 1 and $2 \mathrm{mM}$ (Lusk et al. 1968; Alatossava et al. 1985). It is possible that magnesium concentration plays a role in the global regulation of the initiation mechanism at translational expression by fine tuning the order of events during initiation.

\section{TRANSITION TO ELONGATION}

The final key event that marks the end of initiation is the release of initiation factors and arrival of the first elongator tRNA encoded by the second codon on the mRNA. Yet the relative timing of IF2 release, 50S subunit joining, and elongator tRNA binding are not known. To 
monitor these events in real time, Tsai et al. (2012) delivered Cy5-IF2, Cy3.5-50S, and Phe(Cy2)tRNA ${ }^{\text {Phe }}$ in a ternary complex with EF$\mathrm{Tu}$ and GTP to $30 \mathrm{~S}$ PICs loaded with fMet(Cy3)tRNA ${ }^{\text {fMet }}$, simultaneously tracking four different labeled components. An IF2 signal was followed by rapid and stable 50S joining. Only IF2 with GTP yielded stable tRNA-bind- ing post 50S subunit joining, whereas IF2 with GDPNP, a nonhydrolyzable analog of GTP, only results in brief tRNA sampling with no stable binding. Postsynchronizing to the departure of IF2 revealed a noticeable overlap between the IF2 and 50S signals (Fig. 2). This 2 sec overlap time of IF2 and 50S occupancy on the 30S PIC was independent of $50 \mathrm{~S}$ concentration,

A

A IF2 + 50S + Tu:Phe-tRNA ${ }^{\text {Phe }}:$ GTP
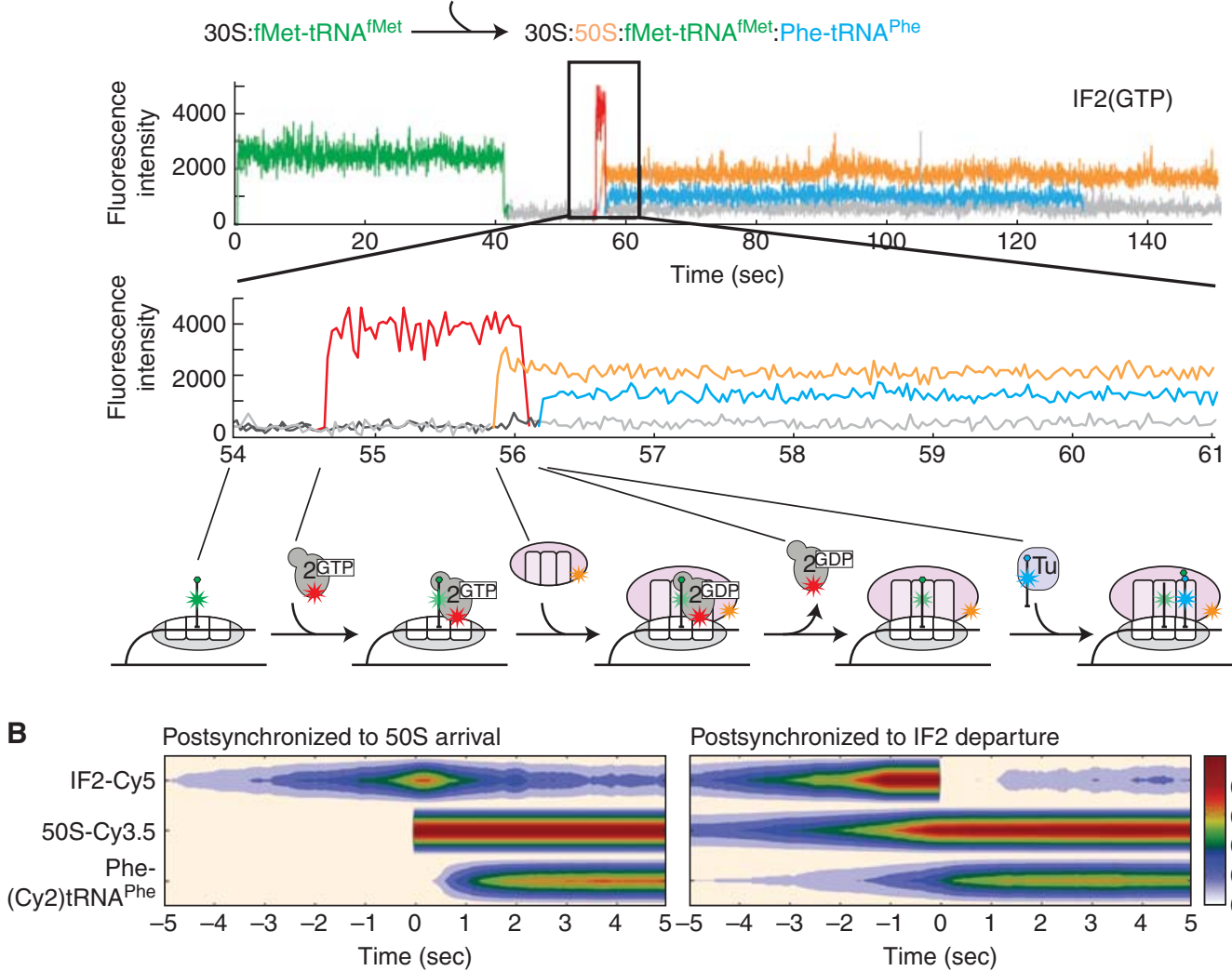

Postsynchronized to IF2 departure

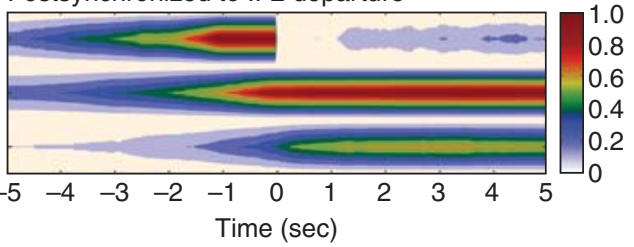

Figure 2. Transition into elongation. (A) Example trace showing the observed order of the late events during initiation. 30S PICs were immobilized via the mRNA in zero-mode waveguide (ZMW) wells and scored by Cy3labeled fMet-tRNA ${ }^{\mathrm{fMet}^{2}}$. Cy3.5-labeled 50S subunit, Cy2-labeled Phe-tRNA ${ }^{\text {Phe }}$ in a ternary complex with EF$\mathrm{Tu}(\mathrm{GTP})$, and Cy5-labeled IF2 were then delivered to the 30S PIC. The disappearance of the IF2 signal corresponds to IF2 dissociation from the 70 S complex, as evidenced by the IF2 residence time that is much shorter than the photobleaching lifetime of the Cy5 dye. The disappearance of all other signals is due to dye photobleaching as indicated by their long photobleaching-limited lifetimes. (B) Postsynchronized plots of IF2, 50S subunit, and elongator tRNA. In the left panel, all single-molecule traces were postsynchronized to the arrival of 50 S, with arrival events defining a new $t=0$ and the right panel is postsynchronized to the departure of IF2 from the $70 \mathrm{~S}$ ribosome. The presence of a signal from the molecule of interest may be read lengthwise, with the color reflecting the mean occupational density at each time point. The left panel shows an $\sim 2$ sec overlap between the IF2 and 50S signals along with a delay in elongator tRNA signal, with most tRNAs arriving predominantly after IF2 release. The right panel shows the heterogeneity of elongator tRNA arrival. In approximately $20 \%$ of the cases, tRNA arrives before IF2 leaves the ribosome. 
A. Petrov et al.

suggesting that a unimolecular process occurs during the overlap. During this period, IF2 rapidly hydrolyzes GTP, rearranges itself, tRNA, and ribosome conformation, and dissociates from the ribosome; consistent with this interpretation, the lifetime of IF2-GDP on 70S ribosomes was $1.2 \mathrm{sec}$. Elongator tRNA arrival required GTP hydrolysis by IF2 and was drastically decreased in the presence of GDPNP. The majority of tRNA (80\%) arrived after IF2 departure. In these subsets of molecules, similar to the early kinetic studies (Tomsic et al. 2000), postsynchronization to the $50 \mathrm{~S}$ arrival time point showed an $\sim 2$ sec lag between 50 S joining and the majority of elongator tRNA arrival. The duration was independent of tRNA concentration $(>200 \mathrm{nM})$, thus indicating that tRNA arrival is a gated unimolecular reaction. The similar duration of the lag and IF2 occupancy time on the ribosome suggests that IF2 release may guide tRNA recruitment. However, the observation that in $20 \%$ of $70 \mathrm{~S}$ subunits, elongator tRNA arrives before IF2 release indicates that IF2 control over tRNA arrival is not absolute.

\section{CLEARING THE SHINE-DALGARNO SEQUENCE}

The Shine-Dalgarno mRNA sequence and 16S rRNA form between five and nine base pairs, with a base-pairing energy of 3-14 kcal $/ \mathrm{mol}$. These interactions must be broken to allow the transition into elongation. The recent structural and single-molecule data provide insight into the potential mechanism for this process (Korostelev et al. 2007).

The mechanical stability of the mRNA-ribosome interactions was examined by optical tweezer assays. In this setup, the mRNA in the translational complexes was immobilized on the surface of the slide and the $30 \mathrm{~S}$ subunit was attached to the polystyrene bead held by the optical trap. The optical trap was used to exert a constantly increasing tension force, until the complexes ruptured. The rupture force required to dislocate mRNA from the ribosome directly reports on the geometry and stability of the mRNA-ribosome interactions. The force required to rupture mRNA from the $70 \mathrm{~S}$ parti- cle depends on the presence of the tRNA ligands and mRNA sequence. The Shine-Dalgarno interaction adds $\sim 10 \mathrm{pN}$, the A-site tRNA $10 \mathrm{pN}$, and the P-site tRNA $\sim 5 \mathrm{pN}$ to the tensile strength of the initiation complexes. However, on formation of the first peptide bond the contribution of the Shine-Dalgarno sequence disappears, suggesting the release of the Shine-Dalgarno-ribosome interaction (Uemura et al. 2007).

\section{DIRECT TRACKING OF THE RIBOSOME MOVEMENT DURING ELONGATION}

The force approach has been used for direct observation of ribosome movement along mRNA. In a breakthrough study, Wen et al. (2008) used a suspended dumbbell assay in which the ends of an mRNA with a hairpin in the center of its sequence are attached to polystyrene beads (hence called "dumbbell') held by dual beam optical tweezers. The optical tweezers allow application of a stretching force sufficient to hold the mRNA in its linear form, permitting accurate measurement of RNA length, as a distance between two trap centers. The translating ribosome unwinds the RNA hairpin and the resulting increase in mRNA length reports on the ribosome position. This was the first direct dynamic observation of ribosome movement along a mRNA at the single-molecule level. Later this approach was expanded to investigate the mechanism of the ribosomal helicase (Qu et al. 2011). The results indicate that the ribosome utilizes two modes of unwinding. First, it promotes and stabilizes the open state of the RNA duplex by sequestering the unwound portion of the duplex. Second, it mechanically unwinds the duplex by pulling on mRNA. The biochemical characterization of the ribosomal helicase activity suggests that proteins S3, S4, and possibly S5 form the helicase center of the ribosome (Takyar et al. 2005) and compose a tight ring around incoming mRNA (Wimberly et al. 2000). These observations are consistent with single-molecule force data and suggest that these proteins may stabilize the open state of the mRNA duplexes or work as an "extrusion die," thus participating in the active mechanism. 
Future studies are needed to show the role of individual ribosomal components in helicase activity and differentiate among various helicase mechanisms.

\section{RIBOSOME CONFORMATIONAL CHANGES DURING INITIATION AND ELONGATION}

Previous cryo-EM methods and structural studies showed that the ribosome adopts two intersubunit conformations-the locked and unlocked states - that are related by a $6^{\circ}$ ratchetlike rotation of the two subunits (Frank and Agrawal 2000; Valle et al. 2003). Peptide bond formation leads to a counterclockwise rotation of the small subunit with respect to the large subunit, from the locked to the unlocked state. Then, eventual GTP hydrolysis and translocation leads to the clockwise locking of the ribosome. These global conformational changes are correlated with the movements of the $\mathrm{L} 1$ protein of the ribosome and the tRNA transitions between the classical state and the hybrid state (Blanchard et al. 2004a,b; Agirrezabala et al. 2008; Fei et al. 2009; Fischer et al. 2010).

Methods to observe ribosome conformation in real time with codon resolution have revealed these dynamic changes directly during translation. Using Cy3-labeled 30S and Cy5-labeled 50S, Marshall et al. characterized an intersubunit FRET signal that reports on the global conformation of the ribosome (Dorywalska et al. 2005; Marshall et al. 2008). The 30S subunit was labeled at the terminal loop of h44 located in the spur of the small subunit, and the 50S subunit was labeled at the loop of h101 placed opposite the central protuberance. The two labeling dyes are separated by $\sim 45 \AA$ according to available structural data, providing a FRET signal that reports on the conformation of the two subunits, yet is distant from the active sites of the ribosome (Fig. 3A).

During the transition from initiation to elongation, IF2 guides the appropriate assembly of the 70S initiation complex on subunit joining. Marshall et al. showed that IF2 accelerates subunit joining, with a subset of ribosomes joining in the rotated low-FRET state. After $\sim 30 \mathrm{msec}$, which agrees with the experimental- ly determined rates for GTP hydrolysis by IF2, the ribosome undergoes a quick transition to the high-FRET state (Marshall et al. 2009). No such transitions were observed with GDPNP. Thus, IF2 GTP hydrolysis guides the ribosome joined in an unproductive low-FRET state into an elongation-competent high-FRET state. However, the sequence of events was not universally observed for every initiating complex. A significant number of ribosomes initiated in the high-FRET (nonrotated) state. The rates of GTP hydrolysis and subsequent intersubunit rotation are comparable with the data acquisition rates, thus it is unclear whether in those molecules conformational changes occurred too fast to be observed, or that the 50S subunits joined in the nonrotated state, resulting in an alternative initiation pathway.

After initiation, the intersubunit FRET signal alternates between a high-FRET state and a low-FRET state. By employing fluorescently labeled tRNA ${ }^{\text {Phe }}$, Aitken et al. (Aitken and Puglisi 2010) directly showed that the transition from high to low FRET occurs concurrently with the arrival of the first elongator tRNA, whereas in the presence of EF-G(GTP), the ribosome then rapidly returns back to the high-FRET (nonrotated) state (Marshall et al. 2008; Aitken and Puglisi 2010). This repeating cycle of highlow-high FRET transitions was observed over multiple rounds of elongation (Aitken and Puglisi 2010). By employing mRNAs of various lengths and withholding a specific tRNA, Aitken et al. observed that the maximum number of high-low-high FRET cycles produced by an elongating ribosome corresponds to the number of codons of the mRNA translated. The addition of the antibiotic erythromycin, which blocks the peptide exit tunnel at a position in which a polypeptide of seven amino acids would reach (Schlunzen et al. 2001), resulted in a significant reduction in the number of ribosomes undergoing translation for more than six FRET cycles (Fig. 3). Thus, a cycle of highlow-high FRET transitions corresponds to one full cycle of elongation. The intersubunit FRET signal provides a method to track multiple elongation cycles and to monitor global conformational dynamics of the ribosome in real time. 
A. Petrov et al.

A

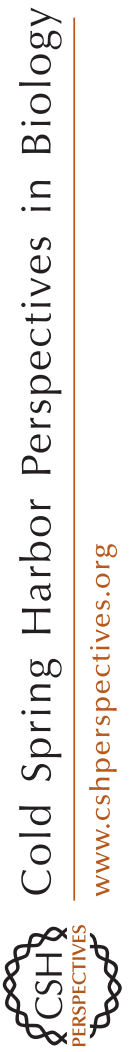

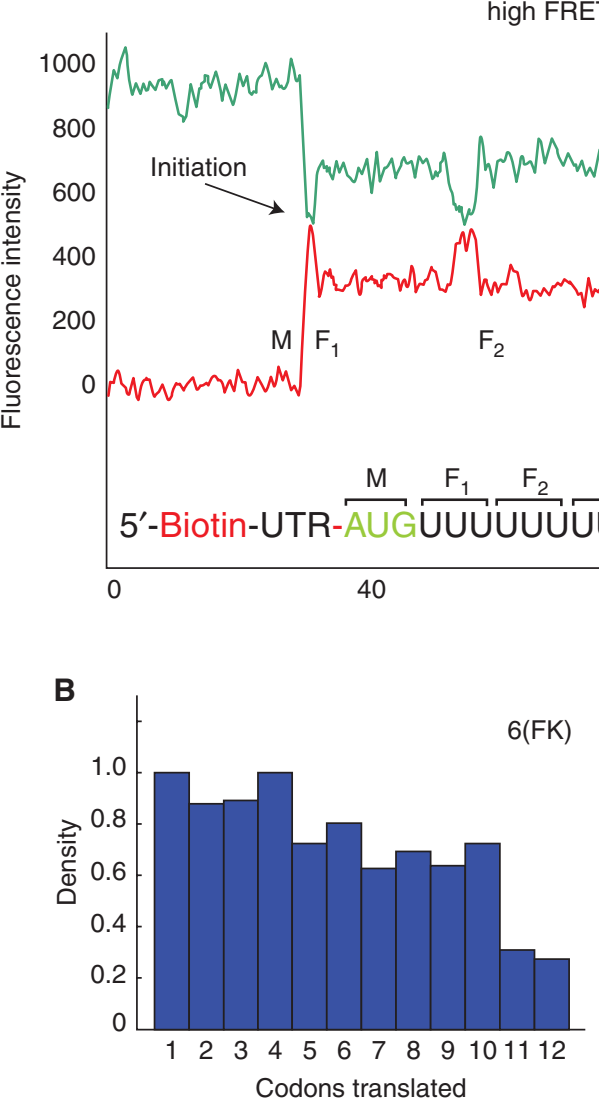

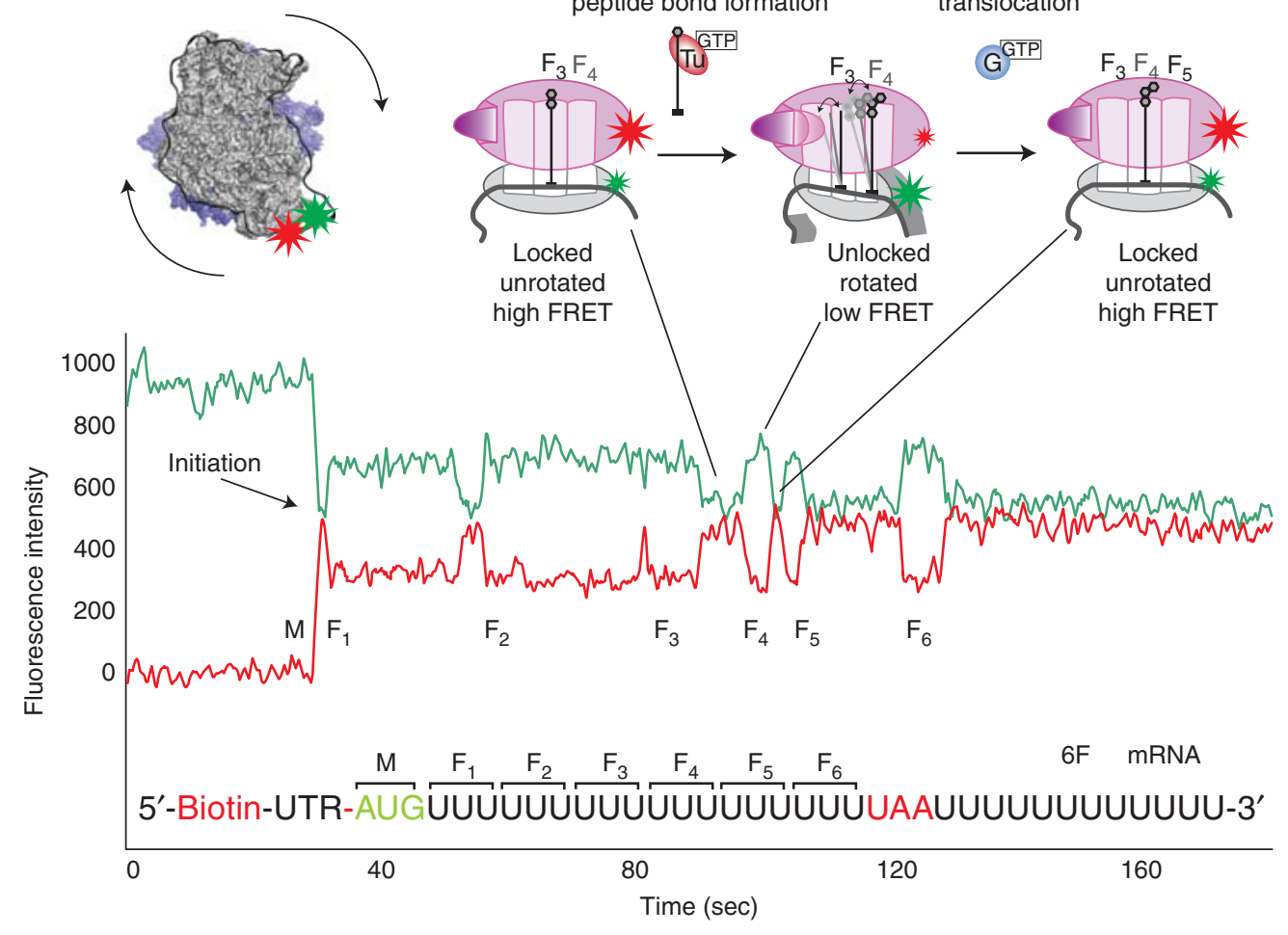

tRNA arrival peptide bond formation
GTP hydrolysis translocation

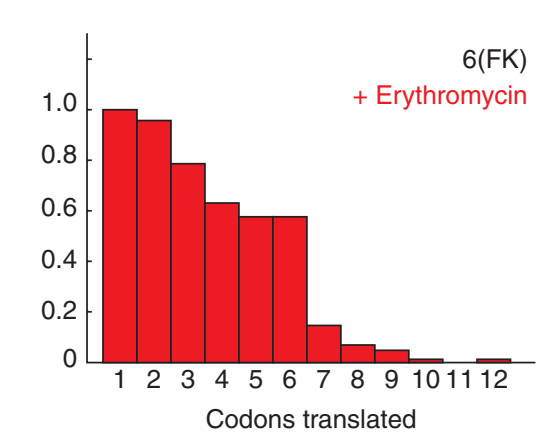

Figure 3. Following global conformation of ribosome during elongation. (A) An intersubunit FRET signal reports on two different rotational states of the ribosome. These states, called "unrotated" and "rotated," have high and low FRET efficiency, respectively. The intersubunit FRET signal allows the observation of changes in global ribosome conformation through multiple rounds of elongation. Cy5-labeled 50S subunits, ternary complexes, and EF-G are delivered to surface-immobilized Cy3-labeled 30S PICs assembled on an mRNA encoding six phenylalanines (designated 6F). The appearance of FRET corresponds to the $50 \mathrm{~S}$ subunit joining during initiation. During elongation, multiple cycles of high-low-high FRETare observed, each corresponding to the ribosome unlocking and locking during one round of elongation. The elongation cycle at the fourth elongator codon is used as an example of high-low-high FRET cycle. (B) Number of FRET cycles observed on mRNAs (designated 6FK) encoding six alternating Phe and Lys pairs. The maximum number of elongation cycles is consistent with the number of codons of the mRNA. Erythromycin stalls ribosomes by blocking the nascent chain at codon 7, which is exactly what is observed. (Modified, with permission, from Aitken and Puglisi 2010.) 
What drives the ribosomal FRET transition? The observed timing of FRET transitions correlates with ample cryo-EM and X-ray data that show intersubunit rotation, suggesting that it reflects a ratcheting motion of the ribosome (Marshall et al. 2008). Because no spontaneous transitions between two states were observed, it was concluded that they are separated by a large energy barrier. Because transition between the two states is dependent on the arrival of tRNA and EF-G, it is possible that the irreversible transition between the two states is induced by peptide bond formation and GTP hydrolysis by EF-G, indicating that the free energies of peptidyl transfer $\left(\sim-8 \mathrm{kcal} \mathrm{mol}^{-1}\right)$ and GTP hydrolysis $\left(\sim-10 \mathrm{kcal} \mathrm{mol}^{-1}\right)$ are required for the rearrangement of the two subunits.

\section{CORRELATIONS BETWEEN tRNA AND RIBOSOME CONFORMATIONS DURING TRANSLATION}

Translation is a dynamic process that requires the intricate interplay between the ribosome, tRNAs, and multiple factors. The major problem for multicomponent experiments is that the small number of dyes suitable for single-molecule fluorescence experiments limits the number of components that can be observed simultaneously. Chen et al. (2012) used the same labeling strategy as Marshall et al. (2008), but replaced Cy5 as the FRET acceptor with a nonfluorescent, black hole quencher (BHQ). The use of BHQ frees the spectral region of the acceptor dye for labeling other components of the system in multiplexed experiments, so it is possible to use Cy5 to label other translation factors, such as tRNA or elongation factors, whereas fluctuating Cy3 intensity can still be utilized to determine the global conformational dynamics of the ribosome.

Using this approach the authors followed changes in ribosome conformation with $\mathrm{Cy} 3 /$ BHQ-labeled ribosomes and correlated them to tRNA dynamics by employing Cy5-labeled tRNAs. Arrival of Cy5-tRNA, shown as a red fluorescent pulse, was concomitant with the transition from high FRET to low FRET of the ribosome. Thus, the arrival of tRNA occurs si- multaneously with ribosome unlocking, within the time resolution of $100 \mathrm{msec}$ (Chen et al. 2012). Similarly, the departure of tRNA occurs simultaneously with the ribosome locking and translocation, also within the time resolution of $100 \mathrm{msec}$. This shows that tRNA arrival and departure are correlated with the ribosome conformational changes. This method can be further extended to reveal the correlation between the ribosomal conformational changes and factor and tRNA dynamics.

\section{tRNA TRANSIT THROUGH THE RIBOSOME}

tRNAs must be correctly selected and then must transit through the ribosome during translation elongation. Aminoacylated tRNA first arrives in the A site as a ternary complex with EF-Tu-GTP. On tRNA selection, the ribosome catalyzes the formation of the peptide bond between the aminoacylated tRNA and the nascent peptide chain attached to the tRNA in the P site. After the peptidyl-transfer reaction takes place, the ribosome changes to a conformation in which the tRNAs and mRNA are conformationally mobile. EF-G catalyzes translocation, moving the A-site tRNA into the P site and also clearing the A site for the next tRNA. At this stage, the original A-site tRNA is now stably bound in the $\mathrm{P}$ site and this completes one round of elongation. After another round of elongation, the Psite tRNA is moved into the E site, where tRNA eventually dissociates from the E site, completing its life cycle on the ribosome. There are two proposed mechanisms of how tRNA dissociates from the ribosome: either allosterically with the arrival of the next tRNA to the A site while potentially regulating tRNA arrival and selection, or spontaneously as soon as it reaches the E site. Uemura et al. (2010) directly tracked tRNAs labeled with fluorescent dyes in elongation experiments, and observed rapid and spontaneous release of tRNA from the E site. However, in Chen et al. (2011), the investigators reported that both pathways of tRNA release occur. These experiments were conducted at $15 \mathrm{mM}$ magnesium, significantly higher than the physiological concentration, potentially allowing for overstabilizing the E-site tRNA and 
A. Petrov et al.

factor-independent spontaneous translocation of the ribosome. Moreover, the partition between the two mechanisms of tRNA arrival remained constant in the range of tRNA concentrations tested, despite an expected increase in the E-site departure rate for the allosteric pathway with increasing tRNA concentration. On the other hand, at a lower $(5 \mathrm{mM}) \mathrm{Mg}^{2+}$ concentration, Uemura et al. (2010) did not observe a detectable overlap between the $\mathrm{P}$ and E-site tRNA signals even when the ribosome is translocating quickly at $1 \mu \mathrm{M}$ tRNA and elongation factor concentrations, suggesting that tRNAs rapidly and (within $\sim 50 \mathrm{msec}$ ) spontaneously depart from the E site (Fig. 4).

\section{tRNA DYNAMICS IN THE RIBOSOME}

The binding dynamics and conformation of the tRNAs on the ribosome play important roles in the tRNA selection and translocation steps of elongation (Korostelev et al. 2006). At the beginning of an elongation cycle (Fig. 5), the ribosome is in the posttranslocation state, with the P-site tRNA stably bound to the ribosome with little conformational fluctuation. In this state, the entire tRNA is located within the P site of both the small and large subunits. The A site is empty at this stage, awaiting the arrival of the next tRNA. When a tRNA arrives in a ternary complex (TC) with EF-Tu.GTP, the anticodon loop on the tRNA comes into contact with the codon on the mRNA in the small subunit A site.
Here, the tRNA is in the A/T state and initial selection occurs in which the codon-anticodon interaction is checked to determine if the tRNA is cognate to the codon. In the A-site tRNA to Psite tRNA (tRNA-tRNA) FRET experiments conducted by Blanchard et al. (Blanchard et al. 2004a,b), this is observed as a low-FRETefficiency state. tRNAs that are not cognate to the next codon on the mRNA only very briefly sample this state (lifetime less than $50 \mathrm{msec}$ ) and then dissociate from the A site.

In the case of a cognate tRNA, the correct codon-anticodon interactions induce a local conformational change in the A site of the small subunit, destacking two adenines (A1492 and A1493) in the decoding site within helix 44 of the 16S rRNA so that the bases are in an orientation to interact with the anticodon arm of the tRNA (Ogle et al. 2001). These interactions stabilize the tRNA in the A site to allow time for further contact between EF-Tu and the GTPase activation center on the large subunit. On GTPase activation, tRNA is further inserted into the A site, resulting in a higher mediumFRET efficiency (0.50) to the P-site tRNA. Subsequent GTP hydrolysis by EF-Tu destabilizes the EF-Tu on the ribosome and sets the stage for the full accommodation of the tRNA. Subsequently, accommodation offers a final chance to reject the tRNA if it is incorrect. After clearing the accommodation step, the tRNA is fully in the A site of both subunits, detected as a highFRET efficiency (0.75) state. This completes a

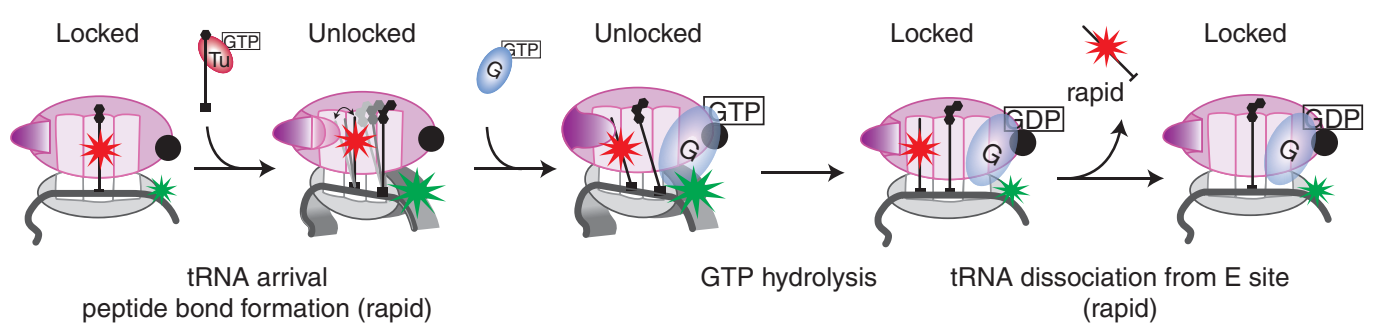

Figure 4. Correlating tRNA occupancy and conformation of the ribosome. tRNA arrives in the A site (corresponds to the appearance of the red fluorescence in the trace above). Peptide bond formation occurs rapidly and the ribosome adopts rotated conformation (low to high FRET transition). Subsequent EF-G catalyzed translocation results in reverse intersubunit rotation (high to low FRET transition) and tRNA movement into the E and P sites, which is followed by rapid dissociation of Cy5 E-site tRNA (disappearance of the red tRNA signal). (Modified, with permission, from Chen et al. 2012.) 


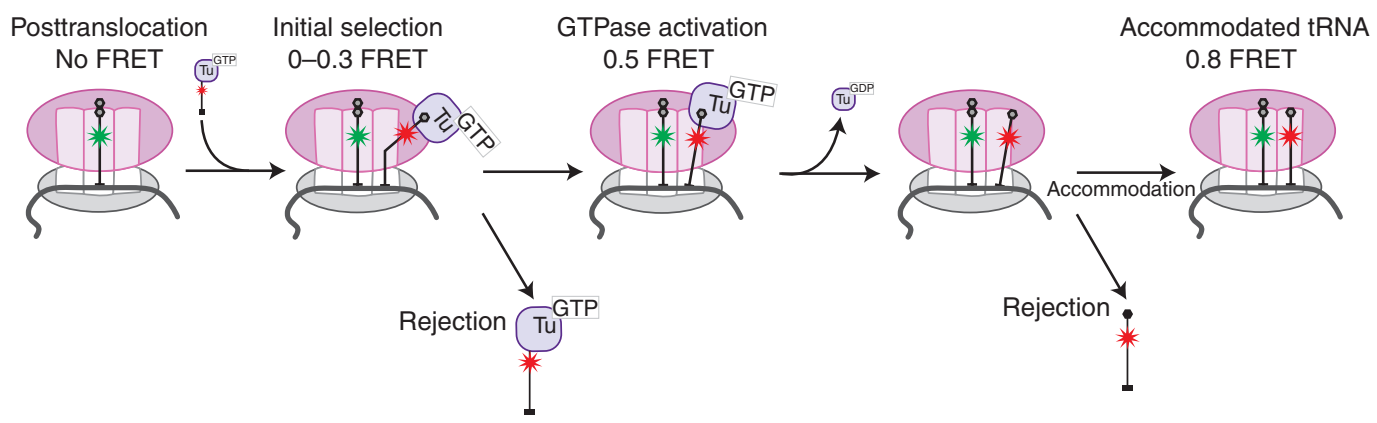

Figure 5. tRNA selection. tRNA arrival to the A site of the small subunit is a multistep process that involves two stages of error checking. FRET value indicated for each stage is from Blanchard et al. (2004b). The tRNA arrives at the A site as a ternary complex with $\mathrm{EF}-\mathrm{Tu}(\mathrm{GTP})$ and adopts a bent A/T conformation. Initial selection takes place through the decoding site of the small subunit A site checking the codon-anticodon interaction and stabilizing the tRNA if the correct interaction is formed. If the tRNA is not rejected, the next stage, GTPase activation, follows. EF-Tu rapidly hydrolyzes GTP, and dissociates from the ribosome. The released acceptor end of tRNA spontaneously moves into the peptidyl transferase center. During this process ribosome has one last chance to check the tRNA for correctness and reject it if it is incorrect. After successful accommodation ribosome is ready to catalyze peptide bond formation between the polypeptide on the P-site tRNA and the amino acid on the A-site tRNA.

tRNA selection, which occurs within $100 \mathrm{msec}$ after initial binding of a cognate tRNA. This two-stage selection mechanism improves selectivity by amplifying the limited increase in tRNA stability from correct codon-anticodon interactions compared with incorrect codonanticodon pairs (Thompson and Stone 1977; Ruusala et al. 1982).

Immediately after tRNA accommodation, both the P-site and the A-site tRNAs are completely bound in their respective ribosomal sites in the classical conformation state (denoted as $\mathrm{A} / \mathrm{A}-\mathrm{P} / \mathrm{P}$, indicating that the tRNAs are completely in their respective sites), with very little tRNA conformation fluctuations (Fig. 6). On peptide bond formation that transfers the polypeptide chain from the P-site to the A-site tRNA, the ribosome unlocks and allows the portions of the tRNA in the large subunit to fluctuate. At this stage, the tRNAs can adopt hybrid conformations in which their anticodon loop is still in its original site in the small subunit but the other parts of the tRNA have moved into the next site in the large subunit (denoted as $\mathrm{A} / \mathrm{P}-\mathrm{P} / \mathrm{E}$ ). Fluctuations in tRNA conformations between the classical state and hybrid states are seen as frequent FRET efficiency fluctuations $\left(2-5 \mathrm{sec}^{-1}\right)$ between the high- and
medium-FRET states. The medium-FRET states likely represent a collection of different tRNA conformations, as Munro et al. (2007) identified at least two distinct hybrid states in subsequent tRNA-tRNA FRET studies. This second identified hybrid state may represent tRNA conformations in which only the P-site tRNA has partially fluctuated into the next site (A/A-P/E).

The dynamic nature of the tRNAs when the ribosome is in the pretranslocation state plays an important role in finding the right conformation for the tRNAs to adopt before translocation mediated by EF-G occurs. As translocation involves shifting the $\mathrm{P}$ - and A-site tRNAs into the next site, having the tRNAs in the $\mathrm{A} / \mathrm{P}-$ $\mathrm{P} / \mathrm{E}$ hybrid state, in which the tRNAs have already partially moved into the next site, likely presents a lower energy barrier. Therefore, the ability for tRNAs in the ribosome to fluctuate and freely explore the hybrid states may have a direct impact on how efficiently translocation occurs. Accordingly, Feldman et al. (2010) reported that addition of antibiotics from the aminoglycoside family that stabilize the classical $(\mathrm{A} / \mathrm{A}-\mathrm{P} / \mathrm{P})$ state results in commensurate reduction in EF-G-catalyzed translocation rates. Members of this family bind to the A site of the small subunit in the decoding site and perturb 
A. Petrov et al.

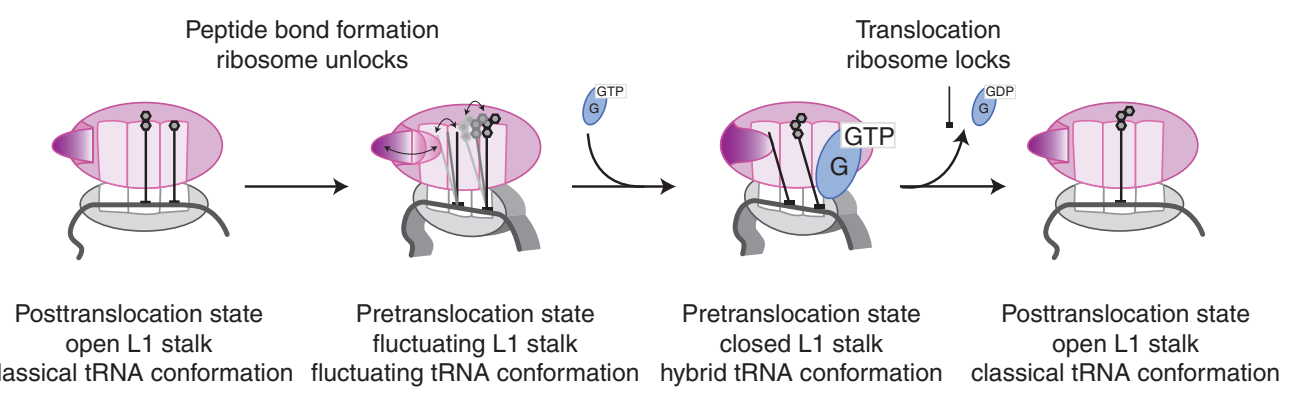

Figure 6. Translocation. The process of ribosome translocation in relation to tRNA dynamics is depicted in the diagram. The flap to the left of the large subunit represents the L1 stalk. The process begins immediately after tRNA accommodation in the A site (as detailed in Fig. 5) in the posttranslocation ribosome state, in which the L1 stalk is open and the tRNAs are in the classical A/A-P/P conformation. On peptide bond formation, the polypeptide chain is transferred to the A-site tRNA and the ribosome unlocks into the pretranslocation state. At this stage, the ribosome and tRNA become conformationally dynamic, with the L1 stalk fluctuating between the open and closed states and the tRNA between the classical conformation (A/A-P/P) and hybrid conformations (A/A-P/E or A/P-P/E). EF-G(GTP) binding locks the L1 stalk into the closed state and the tRNA in the hybrid state (A/P-P/E), readying the ribosome for translocation. On translocation, aided by EF-G, the ribosome locks into a conformationally static state, opening the L1 stalk, releasing the E-site tRNA, and stabilizing the Psite tRNA in the classical conformation.

its local structure, resulting, for many members of the aminoglycoside family, in A1492 and A1493 being destacked regardless if a cognate tRNA is present. This effectively freezes the small subunit A site in a conformation that further stabilizes the classical tRNA conformation. Furthermore, as EF-G interacts with A1492 and A1493 during translocation (Gao et al. 2009), the conformation of helix 44 forced by aminoglycosides may also slow translocation by mechanical opposition. Thus, these effects combine to increase significantly the activation energy barrier for EF-G-mediated translocation.

\section{tRNA-RIBOSOME INTERACTIONS AND TRANSLOCATION}

In addition to the conformational fluctuations of the tRNAs themselves in the ribosome, the Psite tRNA also interacts with the L1 stalk of the large subunit (located near the E site and composed of helices 76-78 of the 23S rRNA and ribosomal protein L1) when it is in a $\mathrm{P} / \mathrm{E}$ configuration. Such an interaction could be central in moving the tRNA when the ribosome translocates. Using FRET between the P-site tRNA and ribosomal protein L1, Fei et al. (2008) reported that the L1 stalk and the P-site tRNA in posttranslocation complexes are relatively static and are distant $(50-70 \AA$, FRET of $0.2-0.4)$ from each other. After peptide bond formation unlocks the ribosome, the L1 stalk and P-site tRNA become dynamic and fluctuate at rates of $1-3 \mathrm{sec}^{-1}$ between the original low-FRET state and a high-FRET state $(0.8$, a distance of $\sim 35 \AA$ ), in which the P-site tRNA in the P/E hybrid conformation is within distance to interact with the L1 stalk. The investigators further observed that EF-G binding to the ribosome shifts all molecules into the hybrid conformation (high-FRET state), maintaining contact until the tRNA is moved into the E site. Thus, tRNA and the ribosome must work in concert in their respective conformational dynamics in order to set the stage for translocation.

Furthermore, studies employing FRET between ribosomal proteins (Cornish et al. 2009; Fei et al. 2009, 2011) observed that the tRNA acylation state in the A and $\mathrm{P}$ sites and the translation state of the ribosome can change the opening and closing dynamics of the L1 stalk. Employing FRET between ribosomal proteins L33-L1 and L9-S6, Cornish et al. (2009) observed that with an acylated P-site tRNA, normally present in the posttranslocation state, the ribosome adopts an open L1 stalk conformation. 
Presumably, this allows the E-site tRNA to dissociate freely from the ribosome after translocation has occurred. Conversely, the L1 stalk is closed when the P-site tRNA is deacylated, which normally is the condition in the pretranslocation ribosome immediately after peptide bond formation has taken place. Fei et al. $(2009,2011)$ also observed the opening of the L1 stalk with FRET between ribosomal proteins L1-L9 when the ribosome is in a posttranslocation state. However, the investigators also observed significantly more fluctuation in the conformation of the L1 stalk compared with the previous study. In the pretranslocation state, the L1 stalk of a majority of ribosomes $(\sim 70 \%)$ is highly dynamic and fluctuates between the open and closed states at rates of $2-4 \mathrm{sec}^{-1}$. Similar to their Psite tRNA to ribosomal protein L1 FRET study, the fluctuations in the pretranslocation complexes are locked into the closed state on EF-G binding if an elongator tRNA is in the P site. On the other hand, if an initiator tRNA occupies the $P$ site, EF-G is unable to lock the L1 stalk and it continues to fluctuate. Mutant initiator tRNAs that mimic elongator tRNAs in flexibility restored the ability of EF-G to lock the L1 stalk before translocation, suggesting that the structural flexibility of the tRNA could play an important part in regulating translocation.

The studies by Cornish et al. (2009) and Fei et al. $(2009,2011)$ detected different levels of L1 stalk fluctuations above when different labeling sites were used; it is not currently clear if the fluctuations observed in these studies represent local conformational dynamics or global changes on the ribosome. As a specific FRET experiment only provides a single relative distance constraint, without further experiments, it is difficult to conclude if the L1 stalk opening and closing and its interactions with the P-site tRNA in the post- and pretranslocation state directly correlate to global conformational changes on the ribosome.

\section{APPLICATIONS TO EUKARYOTIC TRANSLATION}

The single-molecule toolkit developed for the study of prokaryotic translation is in principle immediately portable to eukaryotic translation. Although the technology itself requires little adaptation for this transition, the intrinsic complexity of the eukaryotic translation machinery makes application of single-molecule approaches considerably more difficult than in the case of prokaryotes. This complexity is principally due to (1) the increased number of protein factors associated with eukaryotic ribosomes, (2) the modulatory effects of eukaryotic mRNA structural elements, such as the $5^{\prime}$-cap structure and $3^{\prime}$-poly(A) tail, and mRNA circularization, and (3) the increased number of regulatory events in eukaryotic translation, particularly initiation. These elements greatly increase the number of dynamic events associated with each stage of the translation cycle, as well as making reconstitution of translation in vitro problematic due to the difficulty of isolating individual factors and optimizing experimental conditions.

Notwithstanding these obstacles, a variety of bulk rapid-mixing kinetic studies have been performed recently on reconstituted Saccharomyces cerevisiae translation systems, and these may form a basis for single-molecule experiments. In particular, the work of Lorsch and coworkers has led to the development of a robust eukaryotic in vitro translation system amenable to experiments with fluorescently labeled protein factors (Acker et al. 2007). This system has been used to study the roles of various initiation factors in subunit joining and in cap recognition (Reibarkh et al. 2008; Mitchell et al. 2010; Park et al. 2011). In parallel, novel strategies for the preparation and immobilization of site-specifically labeled yeast ribosomes have provided a platform for transitioning the bulk systems used in the rapid-mixing kinetics studies to single-molecule experiments (Petrov and Puglisi 2010).

Alongside the canonical eukaryotic initiation pathway, factor-independent initiation observed with internal ribosome entry sites (IRESs) found within many viral mRNAs is an attractive entry point into single-molecule studies of biologically and biomedically relevant eukaryotic translation initiation in vitro.

Interpretation of data reporting on conformational dynamics from both bulk and 
A. Petrov et al.

single-molecule studies had been hindered by the absence of a high-resolution structure of the $80 \mathrm{~S}$ eukaryotic ribosome. The X-ray crystal structure of the yeast $80 \mathrm{~S}$ ribosome was reported recently (Ben-Shem et al. 2010, 2011; Klinge et al. 2011). These structural data facilitate not only interpretation of existing biochemical results, but also will allow the design of new experiments. In the single-molecule case, such structural data allows identification of favorable positions for incorporation of fluorescent and other labels to follow ribosomal subunit and intersubunit factor interplay.

\section{CONCLUSIONS}

Single-molecule methods have provided the first direct glimpses of both compositional and conformational dynamics in the ribosome. The methods provide a necessary link between static structure and bulk mechanism, often providing new perspectives on old problems, as illustrated here by studies of initiation and tRNA binding. Continuing improvements in labeling strategies, single-molecule excitation and detection, and data analysis will further deepen the impact of these methods. The challenge will be to unravel the complexity of eukaryotic translation, providing a dynamic view of biological regulation.

\section{ACKNOWLEDGMENTS}

Single-molecule research in the Puglisi group is funded by NIH grants GM51266 and GM099687. We would like to thank Dr. Sotaro Uemura and all members of Puglisi laboratory for helpful discussions.

\section{REFERENCES}

Acker MG, Kolitz SE, Mitchell SF, Nanda JS, Lorsch JR. 2007. Reconstitution of yeast translation initiation. Methods Enzymol 430: 111-145.

Agirrezabala X, Lei J, Brunelle JL, Ortiz-Meoz RF, Green R, Frank J. 2008. Visualization of the hybrid state of tRNA binding promoted by spontaneous ratcheting of the ribosome. Molec Cell 32: 190-197.

Aitken CE, Puglisi JD. 2010. Following the intersubunit conformation of the ribosome during translation in real time. Nat Struct Mol Biol 17: 793-800.
Alatossava T, Jutte H, Kuhn A, Kellenberger E. 1985. Manipulation of intracellular magnesium content in polymyxin B nonapeptide-sensitized Escherichia coli by ionophore A23187. J Bacteriol 162: 413-419.

Antoun A, Pavlov MY, Andersson K, Tenson T, Ehrenberg M. 2003. The roles of initiation factor 2 and guanosine triphosphate in initiation of protein synthesis. EMBO J 22: 5593-5601.

Antoun A, Pavlov MY, Lovmar M, Ehrenberg M. 2006. How initiation factors tune the rate of initiation of protein synthesis in bacteria. EMBO J 25: 2539-2550.

Ban N, Nissen P, Hansen J, Moore PB, Steitz TA. 2000. The complete atomic structure of the large ribosomal subunit at $2.4 \AA$ resolution. Science 289: 905-920.

Ben-Shem A, Jenner L, Yusupova G, Yusupov M. 2010. Crystal structure of the eukaryotic ribosome. Science 330: 1203-1209.

Ben-Shem A, Garreau de Loubresse N, Melnikov S, Jenner L, Yusupova G, Yusupov M. 2011. The structure of the eukaryotic ribosome at 3.0 A resolution. Science 334: 15241529.

Blanchard SC, Gonzalez RL, Kim HD, Chu S, Puglisi JD. 2004a. tRNA selection and kinetic proofreading in translation. Nat Struct Mol Biol 11: 1008-1014.

Blanchard SC, Kim HD, Gonzalez RL Jr, Puglisi JD, Chu S. 2004b. tRNA dynamics on the ribosome during translation. Proc Natl Acad Sci 101: 12893-12898.

Caserta E, Tomsic J, Spurio R, La Teana A, Pon CL, Gualerzi CO. 2006. Translation initiation factor IF2 interacts with the 30 S ribosomal subunit via two separate binding sites. J Mol Biol 362: 787-799.

Chen C, Stevens B, Kaur J, Smilansky Z, Cooperman BS, Goldman YE. 2011. Allosteric vs. spontaneous exit-site (E-site) tRNA dissociation early in protein synthesis. Proc Natl Acad Sci 108: 16980-16985.

Chen J, Tsai A, Petrov A, Puglisi JD. 2012. Non-fluorescent quenchers to correlate single-molecule conformational and compositional dynamics. J Am Chem Soc 134: 57345737.

Cornish PV, Ermolenko DN, Staple DW, Hoang L, Hickerson RP, Noller HF, Ha T. 2009. Following movement of the L1 stalk between three functional states in single ribosomes. Proc Natl Acad Sci 106: 2571-2576.

Dennis PP, Bremer H. 1974a. Differential rate of ribosomal protein synthesis in Escherichia coli B/r. J Mol Biol 84: 407-422.

Dennis PP, Bremer H. 1974b. Macromolecular composition during steady-state growth of Escherichia coli B-r. J Bacteriol 119: 270-281.

Dorywalska M, Blanchard SC, Gonzalez RL, Kim HD, Chu S, Puglisi JD. 2005. Site-specific labeling of the ribosome for single-molecule spectroscopy. Nucleic Acids Res 33: 182-189.

Dunkle JA, Wang L, Feldman MB, Pulk A, Chen VB, Kapral GJ, Noeske J, Richardson JS, Blanchard SC, Cate JH. 2011. Structures of the bacterial ribosome in classical and hybrid states of tRNA binding. Science 332: 981-984.

Fei J, Kosuri P, MacDougall DD, Gonzalez RL Jr. 2008. Coupling of ribosomal L1 stalk and tRNA dynamics during translation elongation. Mol Cell 30: 348-359. 
Fei J, Bronson JE, Hofman JM, Srinivas RL, Wiggins CH, Gonzalez RL Jr. 2009. Allosteric collaboration between elongation factor $\mathrm{G}$ and the ribosomal $\mathrm{L} 1$ stalk directs tRNA movements during translation. Proc Natl Acad Sci 106: $15702-15707$.

Fei J, Richard AC, Bronson JE, Gonzalez RL Jr. 2011. Transfer RNA-mediated regulation of ribosome dynamics during protein synthesis. Nat Struct Mol Biol 18: 1043-1051.

Feldman MB, Terry DS, Altman RB, Blanchard SC. 2010. Aminoglycoside activity observed on single pre-translocation ribosome complexes. Nat Chem Biol 6: 54-62.

Fischer N, Konevega AL, Wintermeyer W, Rodnina MV, Stark H. 2010. Ribosome dynamics and tRNA movement by time-resolved electron cryomicroscopy. Nature 466 : 329-333.

Frank J, Agrawal RK. 2000. A ratchet-like inter-subunit reorganization of the ribosome during translocation. $\mathrm{Na}$ ture 406: 318-322.

Gao YG, Selmer M, Dunham CM, Weixlbaumer A, Kelley AC, Ramakrishnan V. 2009. The structure of the ribosome with elongation factor $\mathrm{G}$ trapped in the posttranslocational state. Science 326: 694-699.

Ha T. 2001. Single-molecule fluorescence resonance energy transfer. Methods 25: 78-86.

Klinge S, Voigts-Hoffmann F, Leibundgut M, Arpagaus S, Ban N. 2011. Crystal structure of the eukaryotic 60S ribosomal subunit in complex with initiation factor 6. Science 334: 941-948.

Korostelev A, Trakhanov S, Laurberg M, Noller HF. 2006. Crystal structure of a 70S ribosome-tRNA complex reveals functional interactions and rearrangements. Cell 126: $1065-1077$.

Korostelev A, Trakhanov S, Asahara H, Laurberg M, Lancaster L, Noller HF. 2007. Interactions and dynamics of the Shine Dalgarno helix in the 70 S ribosome. Proc Natl Acad Sci 104: $16840-16843$.

Kurata S, Weixlbaumer A, Ohtsuki T, Shimazaki T, Wada T, Kirino Y, Takai K, Watanabe K, Ramakrishnan V, Suzuki T. 2008. Modified uridines with C5-methylene substituents at the first position of the tRNA anticodon stabilize U.G wobble pairing during decoding. J Biol Chem 283: 18801-18811.

Levene MJ, Korlach J, Turner SW, Foquet M, Craighead HG, Webb WW. 2003. Zero-mode waveguides for single-molecule analysis at high concentrations. Science 299: $682-$ 686.

Lockwood AH, Chakraborty PR, Maitra U. 1971. A complex between initiation factor IF2, guanosine triphosphate, and fMet-tRNA: An intermediate in initiation complex formation. Proc Natl Acad Sci 68: 3122-3126.

Lockwood AH, Sarkar P, Maitra U. 1972. Release of polypeptide chain initiation factor IF-2 during initiation complex formation. Proc Natl Acad Sci 69: 3602-3605.

Lusk JE, Williams RJ, Kennedy EP. 1968. Magnesium and the growth of Escherichia coli. J Biol Chem 243: 2618-2624.

Majumdar A, Bose KK, Gupta NK. 1976. Specific binding of Excherichia coli chain initiation factor 2 to fMet-tRnafMet. J Biol Chem 251: 137-140.

Marshall RA, Dorywalska M, Puglisi JD. 2008. Irreversible chemical steps control intersubunit dynamics during translation. Proc Natl Acad Sci 105: 15364-15369.
Marshall RA, Aitken CE, Puglisi JD. 2009. GTP hydrolysis by IF2 guides progression of the ribosome into elongation. Mol Cell 35: 37-47.

Milon P, Carotti M, Konevega AL, Wintermeyer W, Rodnina MV, Gualerzi CO. 2010. The ribosome-bound initiation factor 2 recruits initiator tRNA to the 30S initiation complex. EMBO Rep 11: 312-316.

Mitchell SF, Walker SE, Algire MA, Park EH, Hinnebusch AG, Lorsch JR. 2010. The 5'-7-methylguanosine cap on eukaryotic mRNAs serves both to stimulate canonical translation initiation and to block an alternative pathway. Mol Cell 39: 950-962.

Munro JB, Altman RB, O'Connor N, Blanchard SC. 2007. Identification of two distinct hybrid state intermediates on the ribosome. Mol Cell 25: 505-517.

Ogle JM, Brodersen DE, Clemons WM Jr, Tarry MJ, Carter AP, Ramakrishnan V. 2001. Recognition of cognate transfer RNA by the 30S ribosomal subunit. Science 292: 897-902.

Park EH, Walker SE, Lee JM, Rothenburg S, Lorsch JR, Hinnebusch AG. 2011. Multiple elements in the eIF4G1 $\mathrm{N}$-terminus promote assembly of eIF4G1 PABP mRNPs in vivo. EMBO J 30: 302-316.

Petersen HU, Roll T, Grunberg-Manago M, Clark BF. 1979. Specific interaction of initiation factor IF2 of E. coli with formylmethionyl-tRNA f Met. Biochem Biophys Res Commun 91: 1068-1074.

Petrov A, Puglisi JD. 2010. Site-specific labeling of Saccharomyces cerevisiae ribosomes for single-molecule manipulations. Nucleic Acids Res 38: e143.

Qu X, Wen JD, Lancaster L, Noller HF, Bustamante C, Tinoco I Jr. 2011. The ribosome uses two active mechanisms to unwind messenger RNA during translation. $\mathrm{Na}$ ture 475: $118-121$

Reibarkh M, Yamamoto Y, Singh CR, del Rio F, Fahmy A, Lee B, Luna RE, Ii M, Wagner G, Asano K. 2008. Eukaryotic initiation factor (eIF) 1 carries two distinct eIF5-binding faces important for multifactor assembly and AUG selection. J Biol Chem 283: 1094-1103.

Ruusala T, Ehrenberg M, Kurland CG. 1982. Is there proofreading during polypeptide synthesis? EMBO J 1: 741 745.

Schlunzen F, Zarivach R, Harms J, Bashan A, Tocilj A, Albrecht R, Yonath A, Franceschi F. 2001. Structural basis for the interaction of antibiotics with the peptidyl transferase centre in eubacteria. Nature 413: 814-821.

Schuwirth BS, Borovinskaya MA, Hau CW, Zhang W, VilaSanjurjo A, Holton JM, Cate JH. 2005. Structures of the bacterial ribosome at $3.5 \AA$ resolution. Science 310: 827-834.

Spurio R, Brandi L, Caserta E, Pon CL, Gualerzi CO, Misselwitz R, Krafft C, Welfle K, Welfle H. 2000. The C-terminal subdomain (IF2 C-2) contains the entire fMettRNA binding site of initiation factor IF2.J Biol Chem 275: $2447-2454$.

Sundari RM, Stringer EA, Schulman LH, Maitra U. 1976. Interaction of bacterial initiation factor 2 with initiator tRNA. J Biol Chem 251: 3338-3345.

Takyar S, Hickerson RP, Noller HF. 2005. mRNA helicase activity of the ribosome. Cell 120: 49-58. 
A. Petrov et al.

Thompson RC, Stone PJ. 1977. Proofreading of the codonanticodon interaction on ribosomes. Proc Natl Acad Sci 74: $198-202$.

Tomsic J, Vitali LA, Daviter T, Savelsbergh A, Spurio R, Striebeck P, Wintermeyer W, Rodnina MV, Gualerzi CO. 2000. Late events of translation initiation in bacteria: A kinetic analysis. EMBO J 19: 2127-2136.

Tsai A, Petrov R, Marshall A, Korlach J, Uemura S, Puglisi JD. 2012. Heterogeneous pathways and timing of factor departure during translation initiation. Nature (in press).

Uemura S, Dorywalska M, Lee TH, Kim HD, Puglisi JD, Chu S. 2007. Peptide bond formation destabilizes Shine-Dalgarno interaction on the ribosome. Nature 446: 454-457.

Uemura S, Aitken CE, Korlach J, Flusberg BA, Turner SW Puglisi JD. 2010. Real-time tRNA transit on single translating ribosomes at codon resolution. Nature 464: $1012-$ 1017.

Underwood KA, Swartz JR, Puglisi JD. 2005. Quantitative polysome analysis identifies limitations in bacterial cellfree protein synthesis. Biotechnol Bioeng 91: 425-435.

Valle M, Zavialov A, Sengupta J, Rawat U, Ehrenberg M, Frank J. 2003. Locking and unlocking of ribosomal motions. Cell 114: 123-134.

Wen JD, Lancaster L, Hodges C, Zeri AC, Yoshimura SH, Noller HF, Bustamante C, Tinoco I. 2008. Following translation by single ribosomes one codon at a time. $\mathrm{Na}$ ture 452: 598-603.
Wimberly BT, Brodersen DE, Clemons WM Jr, MorganWarren RJ, Carter AP, Vonrhein C, Hartsch T, Ramakrishnan V. 2000. Structure of the $30 \mathrm{~S}$ ribosomal subunit. Nature 407: 327-339.

Wu XQ, RajBhandary UL. 1997. Effect of the amino acid attached to Escherichia coli initiator tRNA on its affinity for the initiation factor IF2 and on the IF2 dependence of its binding to the ribosome. J Biol Chem 272: $1891-$ 1895.

Wu XQ, Iyengar P, RajBhandary UL. 1996. Ribosome-initiator tRNA complex as an intermediate in translation initiation in Escherichia coli revealed by use of mutant initiator tRNAs and specialized ribosomes. EMBO J 15: 4734-4739.

Yusupov MM, Yusupova GZ, Baucom A, Lieberman K, Earnest TN, Cate JH, Noller HF. 2001. Crystal structure of the ribosome at $5.5 \AA$ resolution. Science 292: 883-896.

Yusupova GZ, Yusupov MM, Cate JH, Noller HF. 2001. The path of messenger RNA through the ribosome. Cell 106: 233-241.

Yusupova G, Jenner L, Rees B, Moras D, Yusupov M. 2006. Structural basis for messenger RNA movement on the ribosome. Nature 444: 391-394.

Zhang W, Dunkle JA, Cate JH. 2009. Structures of the ribosome in intermediate states of ratcheting. Science 325: 1014-1017. 


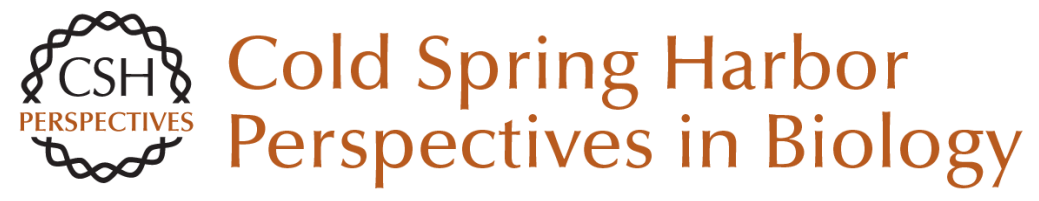

\section{Single-Molecule Analysis of Translational Dynamics}

Alexey Petrov, Jin Chen, Seán O'Leary, Albert Tsai and Joseph D. Puglisi

Cold Spring Harb Perspect Biol 2012; doi: 10.1101/cshperspect.a011551 originally published online July 12,2012

\section{Subject Collection Protein Synthesis and Translational Control}

Tinkering with Translation: Protein Synthesis in Virus-Infected Cells

Derek Walsh, Michael B. Mathews and lan Mohr

Translational Control in Cancer Etiology

Davide Ruggero

A Molecular Link between miRISCs and Deadenylases Provides New Insight into the

Mechanism of Gene Silencing by MicroRNAs Joerg E. Braun, Eric Huntzinger and Elisa Izaurralde

Imaging Translation in Single Cells Using Fluorescent Microscopy Jeffrey A. Chao, Young J. Yoon and Robert $H$. Singer

mRNA Localization and Translational Control in Drosophila Oogenesis Paul Lasko

P-Bodies and Stress Granules: Possible Roles in the Control of Translation and mRNA Degradation Carolyn J. Decker and Roy Parker

Protein Secretion and the Endoplasmic Reticulum Adam M. Benham

From Cis-Regulatory Elements to Complex RNPs and Back

Fátima Gebauer, Thomas Preiss and Matthias W. Hentze
Toward a Genome-Wide Landscape of

Translational Control

Ola Larsson, Bin Tian and Nahum Sonenberg

The Current Status of Vertebrate Cellular mRNA

IRESs

Richard J. Jackson

Principles of Translational Control: An Overview John W.B. Hershey, Nahum Sonenberg and Michael B. Mathews

Regulation of mRNA Translation by Signaling Pathways

Philippe P. Roux and Ivan Topisirovic

The Mechanism of Eukaryotic Translation Initiation: New Insights and Challenges Alan G. Hinnebusch and Jon R. Lorsch

Single-Molecule Analysis of Translational Dynamics Alexey Petrov, Jin Chen, Seán O'Leary, et al.

Cytoplasmic RNA-Binding Proteins and the Control of Complex Brain Function Jennifer C. Darnell and Joel D. Richter

The Elongation, Termination, and Recycling

Phases of Translation in Eukaryotes Thomas E. Dever and Rachel Green

For additional articles in this collection, see http://cshperspectives.cshlp.org/cgi/collection/

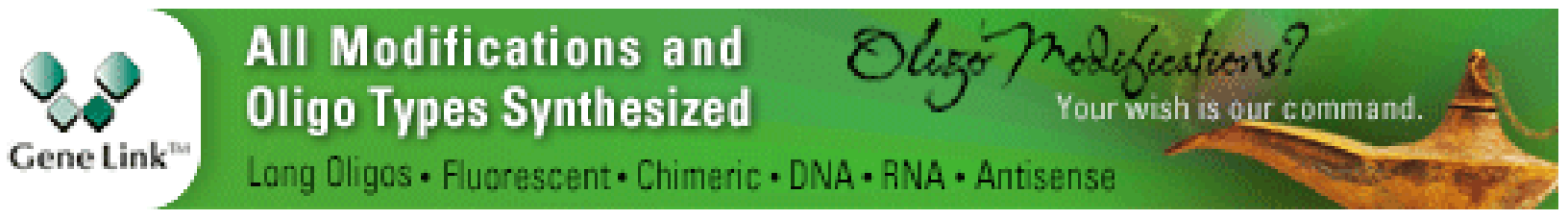


For additional articles in this collection, see http://cshperspectives.cshlp.org/cgi/collection/

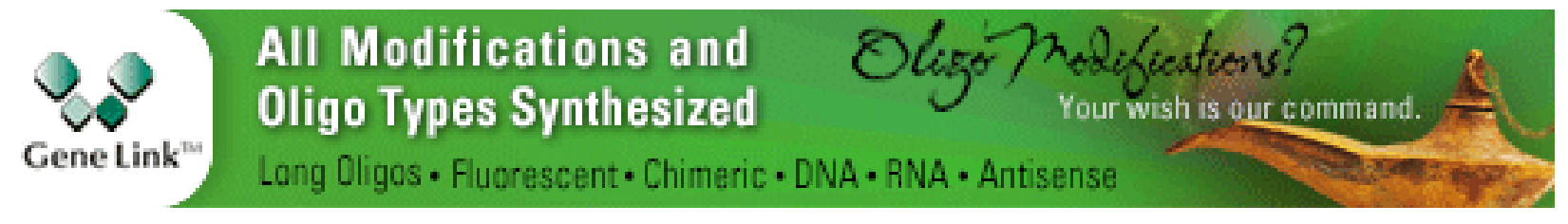

Copyright @ 2012 Cold Spring Harbor Laboratory Press; all rights reserved 